\title{
Polarization effects on Photonic Microwave Generation in VCSELs under Optical Injection
}

Valle, Angel; Quirce, Ana; Ji, Songkun; Hong, Yanhua

\section{IEEE Photonics Technology Letters}

DOI:

10.1109/LPT.2018.2844687

Published: 01/07/2018

Peer reviewed version

Cyswllt i'r cyhoeddiad / Link to publication

Dyfyniad o'r fersiwn a gyhoeddwyd / Citation for published version (APA):

Valle, A., Quirce, A., Ji, S., \& Hong, Y. (2018). Polarization effects on Photonic Microwave Generation in VCSELs under Optical Injection. IEEE Photonics Technology Letters, 30(3), 12661269. https://doi.org/10.1109/LPT.2018.2844687

\footnotetext{
Hawliau Cyffredinol / General rights

Copyright and moral rights for the publications made accessible in the public portal are retained by the authors and/or other copyright owners and it is a condition of accessing publications that users recognise and abide by the legal requirements associated with these rights.

- Users may download and print one copy of any publication from the public portal for the purpose of private study or research.

- You may not further distribute the material or use it for any profit-making activity or commercial gain

- You may freely distribute the URL identifying the publication in the public portal ?
}

Take down policy

If you believe that this document breaches copyright please contact us providing details, and we will remove access to the work immediately and investigate your claim. 


\title{
Polarization effects on Photonic Microwave Generation in VCSELs under Optical Injection
}

\author{
Angel Valle, Ana Quirce, Songkun Ji, and Yanhua Hong, Member, IEEE
}

\begin{abstract}
Photonic microwave generation is studied theoretically in a single-transverse mode VCSEL subject to parallel optical injection. We focus on the dependence of microwave power and frequency on the frequency detuning. We find that, for a fixed injection power, the microwave power at the fundamental frequency is maximum for two different values of the frequency detuning. These results agree qualitatively with those found in recent experiments using optically injected VCSELs. The two maximum microwave powers appear because of the out-of-phase excitation with small amplitudes of both linear polarizations of the VCSEL for frequency detunings between the values at which the two maxima appear.
\end{abstract}

Index Terms - Photonic microwave generation, semiconductor lasers, vertical-cavity surface-emitting lasers, optical injection, radio-over-fiber (RoF), nonlinear dynamics.

$\mathrm{M}$ icrowave photonics has attracted considerable attention recently because of the large demand in radio-over-fiber (RoF) links. By distributing microwave signals over long distances through fibers, the optical carrier of the modulated microwaves has many advantages, such as low cost, high speed, low microwave phase noise, optical single-sideband (SSB) modulation, low power consumption and less system integration complexity [1]. Among the currently used photonic microwave generation techniques, for example, direct modulation [2], optical heterodyne technique [3], external modulation [4], mode-locked semiconductor laser [5], optoelectronic oscillator (OEO) [6] and period one (P1) [7-8], the P1 technique stands out due to having many superior properties [7]. An injected laser in P1 oscillation state can generate a broadly tunable microwave signal frequency far exceeding its relaxation resonance frequency without any microwave input. Also, other advantages of microwave generation based on P1 include SSB modulation realization [7] and low cost due to the all-optical components configuration. Photonic microwave generation based on P1 in distributed

Manuscript received January 29, 2018. This work was supported in part by the Ministerio de Economía y Competitividad (MINECO/FEDER, UE), Spain, under project TEC2015-65212-C3-1-P, and by the Sêr Cymru National Research Network in Advanced Engineering and Materials, (NRN158).

A. Valle is with the Instituto de Física de Cantabria (CSIC-Univ. de Cantabria), Avda. Los Castros s/n, E39005 Santander, Spain (e-mail:valle@ifca.unican.es).

A. Quirce is with the Faculty of Engineering Sciences, Vrije Universiteit Brussel, Brussels 1050, Belgium (e-mail: aquirce@b-phot.org).

$\mathrm{S}$. Ji, and Y. Hong are with the School of Electronic Engineering, Bangor University, Bangor LL57 1UT, U.K. (e-mail: eep40a@bangor.ac.uk; y.hong@bangor.ac.uk ). feedback (DFB) lasers achieving $100 \mathrm{GHz}$ microwave frequency have been reported [8].

Such research interests have also extended to a cost-efficient type of laser: vertical-cavity surface-emitting lasers (VCSELs) [9-11]. Besides the low production cost, VCSELs also offer desirable characteristics, for instance, low threshold current, circular output-beam profile, single-longitudinal mode operation, wafer-scale integrability and longevity. A widely continuously tunable photonic microwave generation based on P1 in an optical injection VCSEL has been demonstrated recently [11]. In [11], two local maximum microwave powers at the two detuning frequencies at a fixed injection power for the higher injection power region have been observed, which has not been shown in DFB lasers and QD lasers. To our knowledge, this phenomenon has not been explained yet.

In this paper, we numerically simulate the photonic microwave generation based on P1 in a VCSEL subject to parallel optical injection. In order to gain insight on the role played by the VCSEL polarization on the dynamics, we perform simulations for VCSELs with low and high values of the linear dichroism. The results show that two maximum microwave powers at the fixed injection power are due to the excitation of both linear polarizations with small amplitudes and with out-of-phase dynamics.

Our theoretical analysis is based on the spin-flip model that describes the dynamical evolution of the polarization modes of a single-transverse mode VCSEL, complemented with an optical injection term. The model and parameters are detailed in [12]. The main laser parameters are the field decay rate, $33 \mathrm{~ns}^{-1}$, the linewidth enhancement factor, 2.8 , the decay rate of the population inversion, $2.08 \mathrm{~ns}^{-1}$, and the linear dichroism $\gamma_{a}=-0.21 \mathrm{~ns}^{-1}$. These parameters have been extracted in [13-14] for a VCSEL that is similar to that used in the experiments of [11]. These VCSELs are commercial devices (Raycan) emitting at 1550 -nm wavelength. We consider also in our simulations the specific characteristics of the laser in [11] with respect to the laser in [12]: for large bias currents the VCSEL in [11] emits in a linear polarization (x) with a frequency that is 5.1 $\mathrm{GHz}$ higher than that of the orthogonal polarization (y), so we take in our model the linear birefringence parameter $\gamma_{p}=-16$ $\mathrm{ns}^{-1}$ corresponding to this situation. As in [11] we consider parallel optical injection in which the direction of the linearly polarized optical injection coincides with that of the linear polarization (x) in which the free-running VCSEL emits. In our calculations we fix the bias current to a value similar to that of [11], that is 6.5 times threshold, and the amplitude of the field of the injected light, $E_{i n j}=1$. 
Fig. 1(a) displays the microwave power as a function of the frequency detuning, defined as the difference between the frequency of the injected light and the frequency of the free-running x-polarization mode [12]. This is the microwave power at the fundamental frequency that corresponds to the total power emitted in both linear polarizations. Two maximum microwave powers are observed at frequency detunings of 1.5 and $6 \mathrm{GHz}$. A trough is observed at $3.45 \mathrm{GHz}$. In the range of detuning of $2-5 \mathrm{GHz}$, both polarizations are excited, but they are not in phase, as we will show later on. This situation is similar to that illustrated in Fig. 5 of [11] at large values of the injection power.

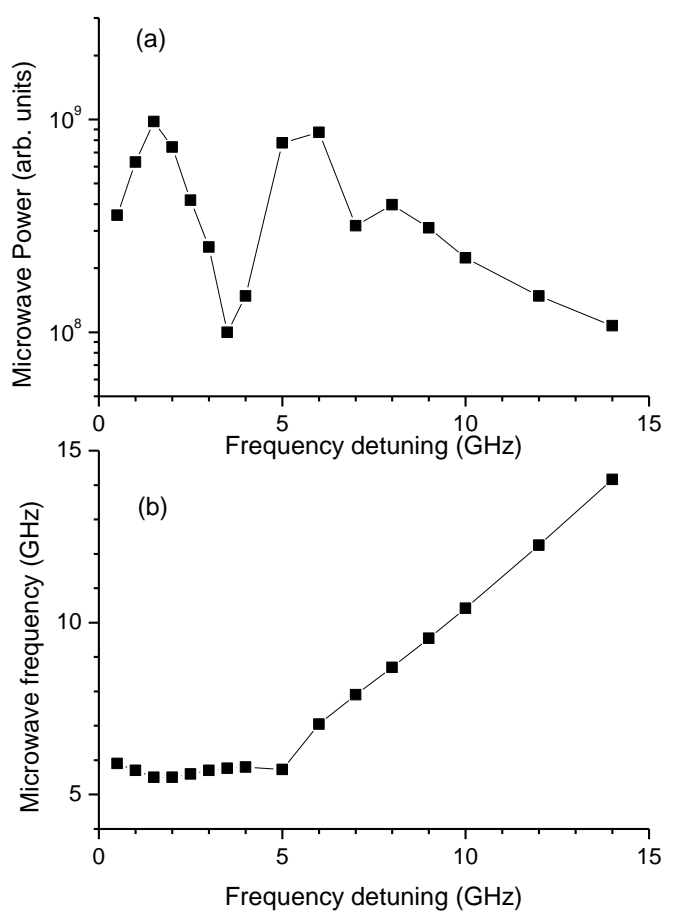

Fig. 1. (a) Generated microwave power and (b) microwave frequency as a function of the frequency detuning.

We also plot in Fig. 1(b) the generated microwave frequency as a function of the frequency detuning. A similar trend to that shown in Fig. 3(a) of [11] is obtained. The microwave frequency obtained for frequency detunings smaller than $5 \mathrm{GHz}$ is close to the theoretical value of the relaxation oscillation frequency, $5.45 \mathrm{GHz}$. A linear increase, with a slope of 0.9 , is observed for frequency detunings larger than $5 \mathrm{GHz}$. P1 oscillation state for the total power is observed in the points shown in Fig.1. Injection locking in the x-polarization mode is observed for frequency detunings smaller than $0.5 \mathrm{GHz}$. So close to this value a Hopf bifurcation is assumed to occur because P1 dynamics in the x-polarization with frequency close to the relaxation oscillation frequency is observed. P1 oscillation in the x-polarization is maintained up to a frequency detuning of $1.5 \mathrm{GHz}$. Above this value the microwave power decreases with the frequency detuning as shown in Fig. 1(a). This happens because the y-polarization mode also becomes excited with P1 dynamics. This situation is illustrated in Fig. 2 in which the optical and RF spectra are plotted for a frequency detuning of $2.5 \mathrm{GHz}$. The optical spectrum is the field power spectrum. This is calculated from the Fast Fourier Transform of the complex electrical field of the $i$-linear polarization mode, $E_{i}$ [12]. The RF spectrum is the power spectrum corresponding to $\left|E_{i}\right|^{2}$. Fig. 2(a) shows that both polarizations are excited with periodic dynamics. Each of these polarizations produces independently a RF signal (at $5.6 \mathrm{GHz}$ in Fig. 2(b)) because that is the frequency separation between consecutive peaks in the optical spectrum of each polarization $(5.6 \mathrm{GHz}$ in Fig. 2(a)). Lines also appear in the RF spectrum corresponding to harmonics of the fundamental frequency. Additional weaker lines appear due to the weaker lines observed in the optical spectrum.

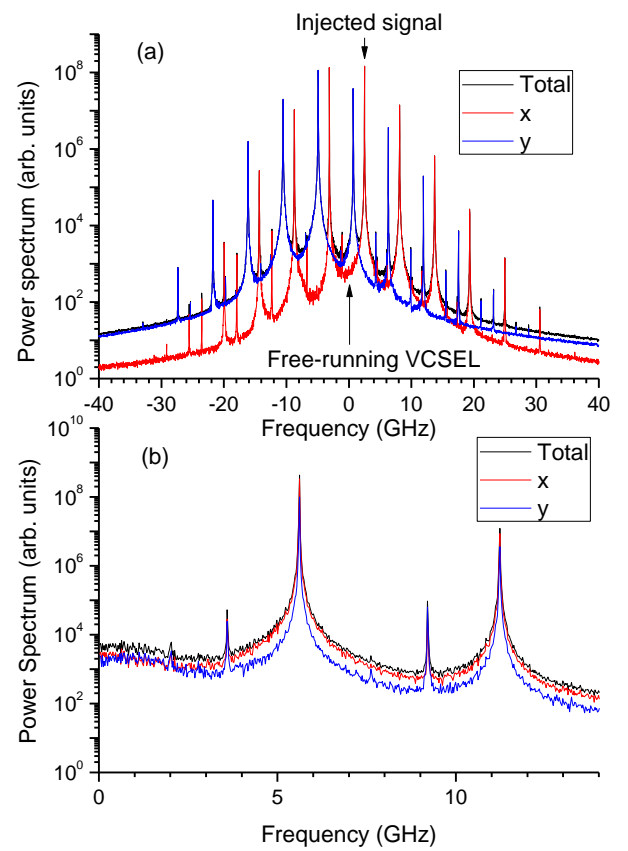

Fig. 2. (a) Optical spectrum and (b) RF spectrum for $2.5 \mathrm{GHz}$ frequency detuning.

Excitation of P1 oscillations in both polarization modes is observed up to $6 \mathrm{GHz}$, that is the frequency detuning value at which the second maximum in the microwave power is obtained. This is shown in Fig. 3 in which maxima of RF spectra for both polarizations are plotted as a function of the frequency detuning. For frequency detunings larger than $6 \mathrm{GHz}$ the VCSEL only emits in the x-polarization. In this range the microwave power (frequency) decreases (increases) with the frequency detuning as it was shown analytically in [15] in which single-mode optically injected semiconductor lasers for microwave generation were studied. This is in agreement with our results for the total power shown in Fig. 1.

The P1 dynamics in both linear polarizations illustrated in Fig. 2 has been experimentally observed in [16]. This dynamics, termed as P1-both, was observed for a VCSEL with a much higher value of the birefringence, $\gamma_{p}=103.1 \mathrm{~ns}^{-1}$. Our results are in agreement with the experiments because the frequencies of the oscillations in the P1-both state are close to the relaxation oscillation frequency [16]. 


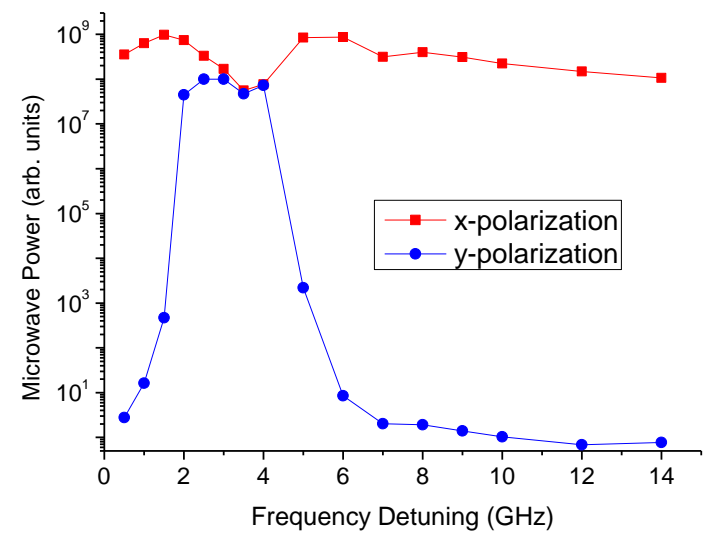

Fig. 3. Generated microwave power for both linear polarization modes.

In order to confirm that the two-peaked structure in Fig. 1(a) is caused by the excitation of both linear polarizations we repeat our simulations for a VCSEL with a much larger value of the linear dichroism. In Figs. 1-3 we considered the value $\gamma_{a}=-0.21 \mathrm{~ns}^{-1}$, similar to that used in [12]. Fig. 4 and Fig. 5 show the results obtained for $\gamma_{a}=-5 \mathrm{~ns}^{-1}$. We have chosen this large value of $\gamma_{a}$ in order to assure single polarization mode emission in all the frequency detuning range. Fig. 4(a) shows that there is only one maximum microwave power, as expected for the single-mode case [15], that is obtained for a frequency detuning close to $3 \mathrm{GHz}$. Fig. 4(b) shows that the microwave frequency increases linearly for frequency detunings larger than $4 \mathrm{GHz}$ and with similar values to those shown in Fig. 1(b), as expected because in both cases there is single-polarization mode emission. This linearly polarized emission is illustrated for a value of the frequency detuning of $2.5 \mathrm{GHz}$ in Fig. 5. In this figure optical and RF spectrum for both linear polarizations are shown.

Fig. 4(b) also shows that for frequency detunings smaller than $4 \mathrm{GHz}$ the microwave frequency decreases with the detuning. This decrease is also observed in Fig. 1(b) while the VCSEL is emitting in a single-polarization (frequency detuning smaller than $1.5 \mathrm{GHz}$ ). However, in the frequency detuning range between 1.5 and $4 \mathrm{GHz}$, results in Fig. 4(b) are different to those in Fig. 1(b) because the emission in the P1-both state is characterized by a microwave frequency close to the relaxation oscillation frequency.

We finally analyze the time traces of the power of both linear polarizations for the cases shown in Fig.5 and Fig. 2, that is for single and two-polarization mode emission. Fig. 6(a) and Fig. 6(b) show the power emitted in both linear polarizations for $\gamma_{a}=-5 \mathrm{~ns}^{-1}$ and $-0.21 \mathrm{~ns}^{-1}$, respectively. Fig. 6(a) shows that almost all the power is emitted in the x-polarization, so the total power and the $\mathrm{x}$-polarized power coincide. The amplitude (frequency) of the oscillation of the total power is larger (smaller) for the single-mode case, in agreement with results shown in Fig. 1 and Fig. 4. Fig. 6(b) shows that oscillations of the power in both polarizations are not in phase. Also the amplitude of the power oscillations of both polarizations is less than half that in the single-mode case.

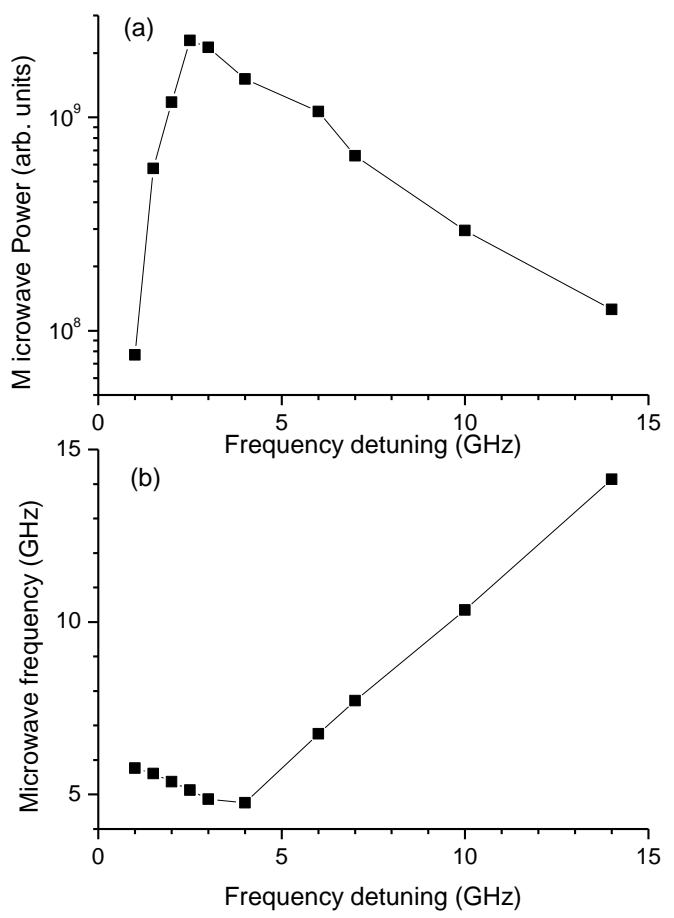

Fig. 4. (a) Generated microwave power and (b) microwave frequency as a function of the frequency detuning. In this figure $\gamma_{a}=-5 \mathrm{~ns}^{-1}$.

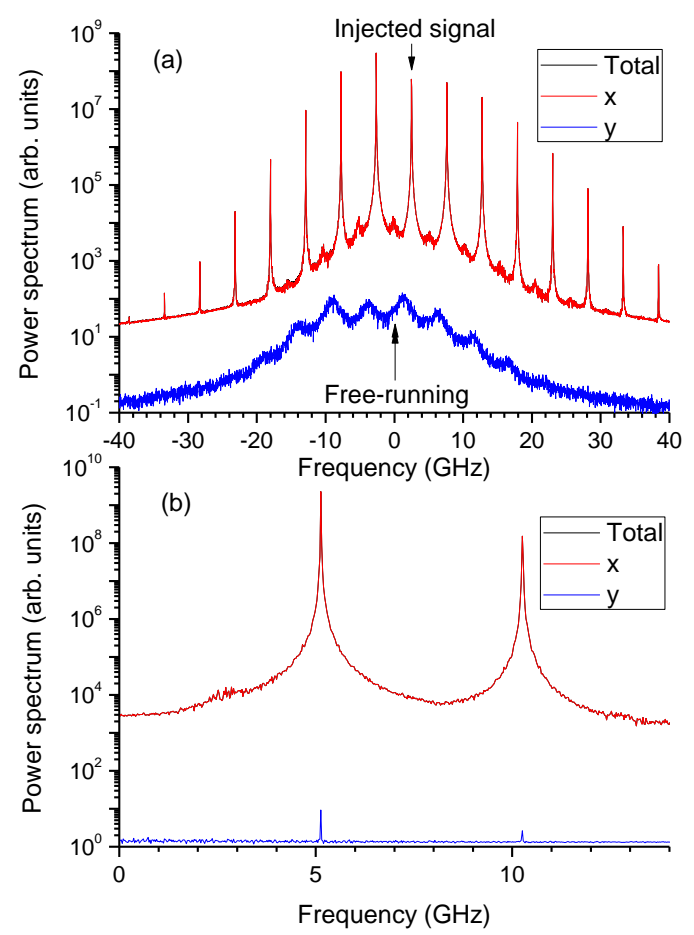

Fig. 5. (a) Optical spectrum and (b) RF spectrum for $2.5 \mathrm{GHz}$ frequency detuning. In this figure $\gamma_{a}=-5 \mathrm{~ns}^{-1}$.

Hence adding both powers in order to get the total power results in oscillations of the total power with smaller amplitude than for the single-mode case. In this way the decrease in the microwave power observed in Fig. 1 when there is two-polarization emission appears because of the combination of the polarizations being out of phase and the amplitude of the 
power oscillations of both polarizations being less than half that in the single-mode case. Our results are in agreement with previous experiments because out-of-phase dynamics was observed in the experimental time series corresponding to P1-both (see Fig. 3(f) and Fig. 4(d) in [16]).

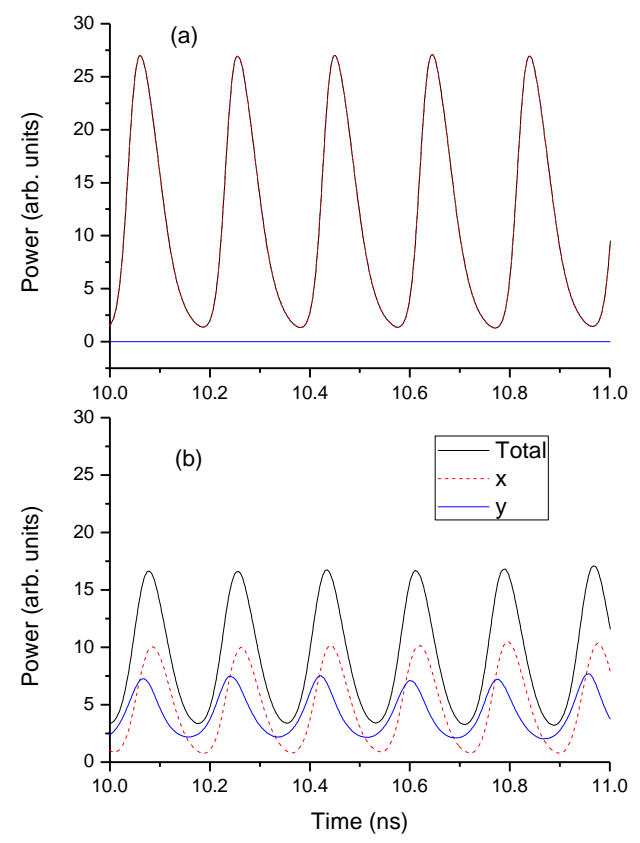

Fig. 6. Time traces of the total power, and the power of both linear polarizations for (a) $\gamma_{a}=-5 \mathrm{~ns}^{-1}$, and (b) $\gamma_{a}=-0.21 \mathrm{~ns}^{-1}$. In this figure the frequency detuning is $2.5 \mathrm{GHz}$.

In conclusion, we have made a theoretical study of the photonic microwave generation based on period-one dynamics of long-wavelength single-transverse mode VCSELs subject to parallel optical injection. We have focused our attention on the microwave power and frequency corresponding to the total power emitted in both linear polarizations of the VCSEL. We have obtained a dependence on the frequency detuning similar to that experimentally found [11]. The microwave signal can be broadly tunable through the adjustment of the frequency detuning. We have shown that for a fixed injection power, the microwave power at the fundamental frequency is maximum for two different values of the frequency detuning, in agreement with [11]. This two-peaked structure is due to the excitation of both linear polarizations of the VCSEL at frequency detunings between the values at which the two maxima appear. We have found that this period-one dynamics appears with a frequency close to the relaxation oscillation frequency and with out-of-phase dynamics between the power of both linear polarizations, in agreement with recent experiments [16]. The combination of the decrease of the amplitude of the power oscillations of both polarizations and the out-of-phase dynamics is responsible for the decrease of the microwave power that appears when the VCSEL emits in both linear polarizations.

\section{ACKNOWLEDGMENT}

This work has been funded by the Ministerio de Economía y Competitividad (MINECO/FEDER, UE), Spain, under project
TEC2015-65212-C3-1-P and the Ser Cymru National Research Network in Advanced Engineering and Materials (NRN158). A. Quirce acknowledges Fonds voor Wettenschapplijk Onderzoek-Vlaanderen fellowship.

\section{REFERENCES}

[1] J. Yao, "Microwave photonics," J. Lightwave Technol. Vol. 27, no. 3, pp. 314-335, 2009.

[2]O. Kjebon, R. Schatz, S. Lourdudoss, S. Nilsson, B. Stalnacke, and L. Backbom, "30 GHz direct modulation bandwidth in detuned loaded InGaAsP DBR lasers at $1.55 \mu \mathrm{m}$ wavelength," Electron. Lett., vol. 33, pp. 488-489, 1997.

[3]U. Gliese, T. N. Nielsen, S. Norskov, and K. E. Stubkjaer, "Multifunctional fiber-optic microwave links based on remote heterodyne detection," IEEE Trans. Microw. Theory Tech., vol. 46, no. 5, pp. 458-468, May 1998.

[4] J. J. O'Reilly, P. M. Lane, R. Heidemann, and R. Hofstetter, "Optical generation of very narrow linewidth millimetre wave signals," Electron. Lett., vol. 28, pp. 2309-2311, 1992.

[5]A. C. Bordonalli, B. Cai, A. J. Seeds, and P. J. Williams, "Generation of microwave signals by active mode locking in a gain bandwidth restricted laser structure," IEEE Photon. Technol. Lett., vol. 8, no. 1, pp. 151-153, Jan. 1996. [6] S. Pan and J. Yao, "Wideband and frequency-tunable microwave generation using an optoelectronic oscillator incorporating a Fabry-Perot laser diode with external optical injection", Opt. Lett., Vol. 35, no. 11, pp. 1911-1913, Jun. 2010 [7] X.-Q. Qi and J.-M. Liu, "Photonic microwave applications of the dynamics of semiconductor lasers," IEEE J. Sel. Top. Quantum Electron., vol. 17, no. 5, pp. 1198-1211, 2011

[8] S.-C. Chan, S.-K. Hwang, and J.-M. Liu, "Radio-over-fiber AM-to-FM upconversion using an optically injected semiconductor laser," Opt. Lett., vol. 31, no. 15, pp. 2254-2256, Aug. 2006.

[9]P. Perez, A. Quirce, A. Valle, A. Consoli, I. Noriega, L. Pesquera, and I. Esquivias, "Photonic generation of microwave signals using a single-mode VCSEL subject to dual-beam orthogonal optical injection," IEEE Photonics J. vol. 7, no. 1, p. 5500614, 2015.

[10] H. Lin, S. Ourari, T. Huang, A. Jha, A. Briggs, and N. Bigagli, "Photonic microwave generation in multimode VCSELs subject to orthogonal optical injection," J. Opt. Soc. Am. B Vol. 34, no. 11, pp.2381-2389, 2017.

[11] S. Ji, Y. Hong, P. S. Spencer, J. Benedikt, and I. Davies, "Broad tunable photonic microwave generation based on period-one dynamics of optical injection vertical-cavity surface-emitting lasers," Opt. Express, vol. 25, no. 17, p. 19863, Aug. 2017.

[12] F. Denis-le Coarer, A. Quirce, P. Perez, A. Valle, L. Pesquera, M. Sciamanna, H. Thienpont, and K. Panajotov, "Injection locking and polarization switching in a $1550 \mathrm{~nm}$ VCSEL subject to parallel optical injection," IEEE J. Sel. Top. Quantum Electron., vol. 23, no. 6, p. 1800910, Nov. 2017.

[13] P. Perez, A. Valle, I. Noriega, and L. Pesquera, "Measurement of the intrinsic parameters of single-mode VCSELs," J. Lightwave Technol. vol. 32, no. 8, pp.1601-1607, Apr. 2014.

[14] P. Perez, A. Valle, and L. Pesquera, "Polarization-resolved characterization of long-wavelength vertical-cavity surface-emitting laser parameters," J. Opt. Soc. Amer. B, vol. 31, no. 11, pp. 2574-2580, 2014.

[15] S. C. Chan, "Analysis of an optically injected semiconductor laser for microwave generation," IEEE J. Quantum Electron., vol. 46, no. 3, pp. 421-428, Mar. 2010.

[16] F. Denis-le Coarer, A. Quirce, A. Valle, L. Pesquera, M. Sciamanna, H. Thienpont, and K. Panajotov, "Polarization dynamics induced by parallel optical injection in a single-mode VCSEL," Opt. Lett., vol. 42, no. 11, pp. 2130-2133, 2017. 